\title{
THE DIRICHLET PROBLEM FOR A THIN RECTANGLE
}

\author{
by J. K. KNOWLES \\ (Received 20th February 1967)
}

We consider the Dirichlet problem for Laplace's equation in a rectangle with a view toward determining the asymptotic behaviour of the solution for the case in which the width of the rectangle is small in comparison with its length. Although the construction of an explicit representation of the solution is an elementary matter, the resulting formula is inconvenient for present purposes, and we accordingly proceed along different lines.

After formulating the problem suitably in the following section, we derive in $\S 2$ a formal expansion of the solution in powers of the width-to-length ratio $\varepsilon$ of the rectangular domain. This expansion, which involves the Bernoulli polynomials, turns out to provide the asymptotic description of the solution away from the short sides of the rectangle, as we prove in $\S 3$.

While we shall not do so here, it is possible to obtain an asymptotic representation valid uniformly on the closed rectangle by adding to our result the "boundary layer" corrections required along the short sides. The determination of these corrections involves the solution of a Dirichlet problem for a semi-infinite strip.

Boundary value problems in "thin" domains are encountered in various applications, notably in the theory of elasticity. Such problems for second order elliptic equations, while much simpler than those arising in elasticity, are nevertheless prototypes in certain respects. They have been discussed, for example, in $(1,2,3)$. Our purposes in this note are to point out that the present problem is the simplest of these prototypes, and to record the explicit result associated with it.

\section{The boundary value problem}

We require the solution $U(X, Y ; \varepsilon)$ of the differential equation

$$
U_{X X}+U_{Y Y}=0
$$

on the open rectangle $0<X<1,0<Y<\varepsilon$, satisfying the boundary conditions

$$
\begin{array}{ll}
U(0, Y ; \varepsilon)=g(Y / \varepsilon), & 0 \leqq Y \leqq \varepsilon, \\
U(1, Y ; \varepsilon)=0, & 0 \leqq Y \leqq \varepsilon, \\
U(X, 0 ; \varepsilon)=f(X), & 0 \leqq X \leqq 1, \\
U(x, \varepsilon ; \varepsilon)=0, & 0 \leqq X \leqq 1 .
\end{array}
$$

$\dagger$ Subscripts $X, Y$ and $x, y$ indicate partial differentiation. 
The given functions $f$ and $g$ are required to be continuous $\dagger$ on $[0,1]$ and, for simplicity, independent of $\varepsilon$. They are also required to satisfy the conditions

$$
g(0)=f(0), g(1)=f(1)=0,
$$

so as to assure the continuity of the boundary values of $U$.

The existence of a solution $U$ which is twice continuously differentiable on the open rectangle and continuous on its closure is guaranteed, for each $\varepsilon>0$, by standard theorems.

For a "thin" rectangle, $\varepsilon$ is small compared to unity. To avoid the situation in which the domain varies with $\varepsilon$, and to put in evidence the role played by the small parameter, we make the natural change of variables

$$
x=X, \quad y=Y / \varepsilon, \quad u(x, y ; \varepsilon)=U(x, \varepsilon y ; \varepsilon) .
$$

If $\mathscr{R}$ denotes the open square $0<x, y<1$, the boundary value problem becomes

$$
\begin{array}{cc}
\Delta_{\varepsilon} u \equiv \varepsilon^{2} u_{x x}+u_{y y}=0 & \text { on } \mathscr{R}, \\
u(0, y ; \varepsilon)=g(y), & 0 \leqq y \leqq 1, \\
u(1, y ; \varepsilon)=0, & 0 \leqq y \leqq 1, \\
u(x, 0 ; \varepsilon)=f(x), & 0 \leqq x \leqq 1, \\
u(x, 1 ; \varepsilon)=0, & 0 \leqq x \leqq 1 .
\end{array}
$$

In view of the possibility of superposition, no significant loss of generality is involved in the assumption of homogeneous boundary conditions in (10) and (12).

\section{The formal expansion}

Since our interest is in small positive $\varepsilon$, it is natural to inquire whether the differential equation

$$
u_{y y}=0 \text {, }
$$

obtained from (8) by setting $\varepsilon=0$, can provide a useful approximation. There is, in fact, a solution of (13) which satisfies the boundary conditions (11) and (12) at $y=0$ and $y=1$, respectively. It is given by

$$
u=(1-y) f(x) \text {. }
$$

Viewed as an approximate solution of the boundary value problem (8)-(12), (14) must certainly be of poor quality near the ends $x=0$ and $x=1$, since it will not in general satisfy the boundary conditions imposed there.

An obvious way to generalise the "approximation" (14) (without considering the corrections which will prove to be necessary along the edges $x=0$ and $x=1$ ) is to expand the solution $u$ in powers of $\varepsilon$ and determine the coefficients successively from the differential equation (8) and the boundary 
conditions (11) and (12), ignoring the boundary conditions (9) and (10). While this programme can be carried out without difficulty, we prefer to proceed in the following purely symbolic way.

We rewrite the differential equation (8) in the form

$$
u_{y y}+\varepsilon^{2} D^{2} u=0
$$

where $D=\partial / \partial x$. The "solution" suggested by $(15)$ is

$$
u=[\cos (\varepsilon y D)] A(x)+[\sin (\varepsilon y D)] B(x),
$$

where $A$ and $B$ are arbitrary functions to be determined. If we formally impose the boundary conditions $u=f$ at $y=0$ and $u=0$ at $y=1$, we find

$$
u=\left\{\frac{\sin [\varepsilon(1-y) \partial / \partial x]}{\sin (\varepsilon \partial / \partial x)}\right\} f(x) .
$$

A formal expansion in powers of $\varepsilon$ is now obtained from (17) by utilising the known Taylor series

$$
\frac{\sin (1-y) z}{\sin z}=\sum_{n=0}^{\infty}(-1)^{n+1} \frac{2^{2 n+1}}{(2 n+1) !} B_{2 n+1}(y / 2) z^{2 n}, \quad|z|<\pi
$$

where $B_{k}(t)$ is the Bernoulli polynomial of degree $k$ given by

$$
B_{k}(t)=\sum_{j=0}^{k} \frac{k ! B_{j}}{j !(k-j) !} t^{k-j}
$$

and the constants $B_{j}$ are the Bernoulli numbers. Equation (18) follows from a consideration of the generating function for the polynomials $B_{k}(t)$; the necessary formulas may be found in (5) and elsewhere.

The formal expansion of $u$ in powers of $\varepsilon$ which comes from (17) upon substitution from (18) with $z$ replaced by $\varepsilon \partial / \partial x$ is given by

$$
u \sim \sum_{n=0}^{\infty}(-1)^{n+1} \frac{2^{2 n+1}}{(2 n+1) !} B_{2 n+1}(y / 2) f^{(2 n)}(x) \varepsilon^{2 n} .
$$

In the following section we shall give meaning to this formula.

The first few Bernoulli polynomials are

$$
B_{0}(t)=1, B_{1}(t)=t-\frac{1}{2}, B_{2}(t)=t^{2}-t+\frac{1}{6}, B_{3}(t)=t^{3}-\frac{3}{2} t^{2}+\frac{1}{2} t .
$$

Some of their properties which we shall use are listed below. (See (5).)

$$
\begin{gathered}
B_{1}(0)=-\frac{1}{2} ; B_{1}\left(\frac{1}{2}\right)=0 ; B_{2 k+1}(0)=B_{2 k+1}\left(\frac{1}{2}\right)=0, k=1,2, \ldots ; \\
B_{k}^{\prime}(t)=k B_{k-1}(t), \quad k=1,2, \ldots
\end{gathered}
$$

If only the first term is retained on the right side of (20), we recover (14):

$$
u \sim(1-y) f(x)
$$

if the first two terms are retained, (24) is replaced by

$$
u \sim(1-y) f(x)+\left(\frac{y}{3}-\frac{y^{2}}{2}+\frac{y^{3}}{6}\right) \varepsilon^{2} f^{\prime \prime}(x) .
$$


A similar symbolic calculation can be applied to the Neumann problem for Laplace's equation in a rectangle, and the resulting formal expansion involves the Euler polynomials. While in the case of the Dirichlet problem treated here the boundary conditions at $x=0$ and $x=1$ do not influence the expansion (20), this is not the case for the Neumann problem.

\section{The asymptotic nature of the expansion}

We now prove a theorem which describes the relation of the formal expansion (20) to the exact solution $u(x, y ; \varepsilon)$.

Theorem. In the boundary value problem (8)-(12), let

$$
g \in C^{\prime}[0,1], f \in C^{2 m+2}[0,1],
$$

where $m$ is a fixed nonnegative integer. Let $f$ and $g$ satisfy (6). Then as $\varepsilon \rightarrow 0$,

$$
u(x, y ; \varepsilon)=\sum_{n=0}^{m}(-1)^{n+1} \frac{2^{2 n+1}}{(2 n+1) !} B_{2 n+1}(y / 2) f^{(2 n)}(x) \varepsilon^{2 n}+O\left(\varepsilon^{2 n+2}\right),
$$

uniformly in $(x, y)$ for $\delta \leqq x \leqq 1-\delta, 0 \leqq y \leqq 1$, for any $\delta \in\left(0, \frac{1}{2}\right)$.

In the proof we make use of two preliminary results, the first of which is a consequence of the maximum principle for second order elliptic operators. Let $v$ be continuous on the closure $\overline{\mathscr{R}}$ of $\mathscr{R}$, twice continuously differentiable on $\mathscr{R}$ and a solution of the differential equation

$$
\Delta_{\varepsilon} v=F \quad \text { on } \mathscr{R},
$$

where $F$ is continuous on $\overline{\mathscr{R}}$. If $v$ vanishes on the boundary of $\mathscr{R}$, then

$$
|v| \leqq \max _{\overline{\mathscr{A}}}|F| \text { on } \overline{\mathscr{R}} \text {. }
$$

This result follows from an argument based on the maximum principle and differing only in minor details from the discussion on pp. 329-331 of (4).

The second result which we require refers to the following boundary value problem. Let $w$ be continuous on $\overline{\mathscr{R}}$, twice continuously differentiable on $\mathscr{R}$ and such that

$$
\left.\begin{array}{l}
\Delta_{\varepsilon} w=0 \text { on } \mathscr{R}, \\
w(0, y ; \varepsilon)=G(y ; \varepsilon), \\
w(1, y ; \varepsilon)=w(x, 0 ; \varepsilon)=w(x, 1 ; \varepsilon)=0,
\end{array}\right\}
$$

where $G$ is a continuously differentiable function of $y$ for each $\varepsilon>0$. Then

$$
|w(x, y ; \varepsilon)| \leqq 5 \max |G| \frac{e^{-\pi x / \varepsilon}}{1-e^{-\pi x / \varepsilon}}, \quad 0<x \leqq 1, \quad 0 \leqq y \leqq 1 .
$$

This inequality follows from an elementary estimate based on the Fourier series representation of the explicit solution of the boundary value problem (29). We omit the detailed proof. 
Turning now to the proof of the theorem, we introduce the abbrevatiation

$$
u^{(2 m)}(x, y ; \varepsilon)=\sum_{n=0}^{m}(-1)^{n+1} \frac{2^{2 n+1}}{(2 n+1) !} B_{2 n+1}(y / 2) f^{(2 n)}(x) \varepsilon^{2 n}
$$

for the truncation of (20). The properties (22) of the Bernoulli polynomials show that, for every $m \geqq 0, u^{(2 m)}$ satisfies the boundary conditions (11), (12) imposed on $u$ :

$$
u^{(2 m)}(x, 0 ; \varepsilon)=f(x), \quad u^{(2 m)}(x, 1 ; \varepsilon)=0 .
$$

Property (23) enables us to compute

$$
\begin{aligned}
\Delta_{\varepsilon} u^{(2 m)} & =(-1)^{m-1} \frac{2^{2 m+1}}{(2 m+1) !} B_{2 m+1}(y / 2) f^{(2 m+2)}(x) \varepsilon^{2 m+2} \\
& \equiv-F(x, y ; \varepsilon) .
\end{aligned}
$$

With the notation

$$
U=u-u^{(2 m)},
$$

we conclude from (8)-(12), (31) and (32) that $U$ is a solution of the following boundary value problem:

$$
\begin{aligned}
& \Delta_{\varepsilon} U=F \text { on } \mathscr{R}, \\
& U(x, 0 ; \varepsilon)=U(x, 1 ; \varepsilon)=0, \\
& U(0, y ; \varepsilon)=G_{1}(y ; \varepsilon), \quad u(1, y ; \varepsilon)=G_{2}(y, \varepsilon),
\end{aligned}
$$

where

$$
G_{1}(y ; \varepsilon)=g(y)-u^{(2 m)}(0, y ; \varepsilon), \quad G_{2}(y ; \varepsilon)=-u^{(2 m)}(1, y ; \varepsilon) .
$$

We decompose the problem for $U$ into three simpler problems by setting

$$
U=v+w_{1}+w_{2}
$$

where $v, w_{1}$ and $w_{2}$ are defined by the following Dirichlet problems:

$$
\left.\begin{array}{rl}
\Delta_{\varepsilon} v=F & \text { on } \mathscr{R}, \\
v=0 & \text { on the boundary of } \mathscr{R},
\end{array}\right\}
$$

$$
\Delta_{\varepsilon} w_{1}=0 \text { on } \mathscr{R} \text {, }
$$$$
w_{1}(x, 0 ; \varepsilon)=w_{1}(x, 1 ; \varepsilon)=0 \text {, }
$$$$
w_{1}(0, y ; \varepsilon)=G_{1}(y ; \varepsilon), \quad w_{1}(1, y ; \varepsilon)=0 \text {, }
$$

$\Delta_{\varepsilon} w_{2}=0$ on $\mathscr{R}$,

$w_{2}(x, 0 ; \varepsilon)=w_{2}(x, 1 ; \varepsilon)=0$,

$w_{2}(0, y ; \varepsilon)=0, \quad w_{2}(1, y ; \varepsilon)=G_{2}(y ; \varepsilon)$. 
It is now only necessary to observe that the estimate (28) applies to the solution $v$ of (38), so that, bearing in mind the definition (33) of $F$, we have

$$
|v| \leqq K_{m} \varepsilon^{2 m+2} \text { on } \mathscr{R},
$$

where

$$
K_{m}=\frac{2^{2 m+1}}{(2 m+1) !} \max _{\bar{g}}\left|B_{2 m+1}(y / 2) f^{(2 m+2)}(x)\right| .
$$

The estimate (29) applies to the solution $w_{1}$ of (39), yielding

$$
\left|w_{1}(x, y ; \varepsilon)\right| \leqq 5\left(\max \left|G_{1}\right|\right) \frac{e^{-\pi x / \varepsilon}}{1-e^{-\pi x / \varepsilon}}, \quad 0<x \leqq 1, \quad 0 \leqq y \leqq 1 .
$$

The transformation $x \rightarrow 1-x$ allows us to apply (29) to the solution $w_{2}$ of (40) to obtain

$$
\left|w_{2}(x, y ; \varepsilon)\right| \leqq 5\left(\max \left|G_{2}\right|\right) \frac{e^{-\pi(1-x) / e}}{1-e^{-\pi(1-x) / \varepsilon}}, 0 \leqq x<1, \quad 0 \leqq y \leqq 1 .
$$

Since $G_{1}$ and $G_{2}$ are polynomials in $\varepsilon$, we conclude from (37), (41), (42) and (43) that

$$
U(x, y ; \varepsilon)=O\left(\varepsilon^{2 m+2}\right) \text { as } \varepsilon \rightarrow 0,
$$

uniformly in $(x, y)$ for $\delta \leqq x \leqq 1-\delta, 0 \leqq y \leqq 1$, for any $\delta \in\left(0, \frac{1}{2}\right)$. The proof is thus complete.

\section{REFERENCES}

(1) N. Fox, On asymptotic expansions in plate theory, Proc. Royal Soc. London, Ser. A, 278 (1964), 228-233.

(2) D. R. WestBRooK, Applications of asymptotic integration to potential problems for shells, SIAM J. on Appl. Math. 14 (1966), 131-146.

(3) J. K. KNowLes, A Saint-Venant principle for a class of second order elliptic boundary value problems, Zeit. für Angew. Math. und Phys., 18 (1967), 473-490.

(4) R. Courant and D. Hilbert, Methods of Mathematical Physics, vol. II (Interscience Publishers, New York, 1962). 1924).

(5) N. E. NöRLUND, Vorlesungen über Differenzenrechnung (Springer, Berlin,

Division of Engineering and Applied Science

California Institute of Technology

Pasadena, California 